\title{
CONVERGENCE OF FOURIER SERIES
}

\author{
KEVIN STEPHEN STOTTER CUDDY
}

\begin{abstract}
This paper sets out to explore and explain some of the basic concepts of Fourier analysis and its applications. Convolution and questions of convergence will be central. An application to the isoperimetric inequality will conclude the paper.
\end{abstract}

\section{CONTEnTs}

1. Introduction to Fourier Series 1

2. Convolution and Kernels 2

3. Criteria for Convergence 8

4. Isoperimetric Inequality 18

Acknowledgments 19

References 19

\section{Introduction to Fourier Series}

It will be important for the reader to recall Euler's Formula:

$$
e^{i x}=\cos (x)+i \sin (x), \forall x \in \mathbb{R}
$$

Throughout this paper, an "integrable" function should be interpreted as integrable in the Riemann sense as well as bounded.

Definition 1.2. For an integrable, bounded function $f:[a, b] \rightarrow \mathbb{C}$ we can define, for any integer $n$, the $n$th Fourier coefficient

$$
\hat{f}(n)=\frac{1}{b-a} \int_{a}^{b} f(x) e^{-2 \pi i n x /(b-a)} d x .
$$

When there is no question about the function we are referring to we may write $\hat{f}(n)=a_{n}$. From these coefficients we get the Fourier series $S(x)=\sum_{n=-\infty}^{\infty} \hat{f}(n) e^{2 \pi i n x}$ (note that this sum may or may not converge). To denote that a Fourier series is associated to a function $f$ we write

$$
f \sim \sum_{n=-\infty}^{\infty} \hat{f}(n) e^{2 \pi i n x}
$$

Definition 1.3. The $N^{\text {th }}$ partial sum of the Fourier series for $f$, where $N$ is a positive integer, is given by $S_{N}(f)(x)=\sum_{n=-N}^{N} \hat{f}(n) e^{2 \pi i n x / L}$.

Date: DEADLINE AUGUST 26, 2012. 
Example 1.4. Let $f(x)=x$ on $[-\pi, \pi]$ and let $f(x)$ be $2 \pi$-periodic (extended in the normal sense). Then $\hat{f}(n)=\frac{1}{2 \pi} \int_{-\pi}^{\pi} x e^{-i n x} d x=\frac{i(-1)^{n}}{n}, n \neq 0$ or 0 if $n=0$ after applying integration by parts and trigonometric identities. Then the Fourier series is defined as $\sum_{-\infty}^{\infty} \hat{f}(n) e^{i n x}$. For example, the $2^{n d}$ partial sum $S_{2}$ is $\frac{-i}{2} e^{-2 i x}+i e^{-i x}-i e^{i x}+\frac{i}{2} e^{2 i x}$, or $-\sin (2 x)+2 \sin (x)$. Below is a graph comparing this approximation to $f$; the Fourier series is in green while $f$ is in red.

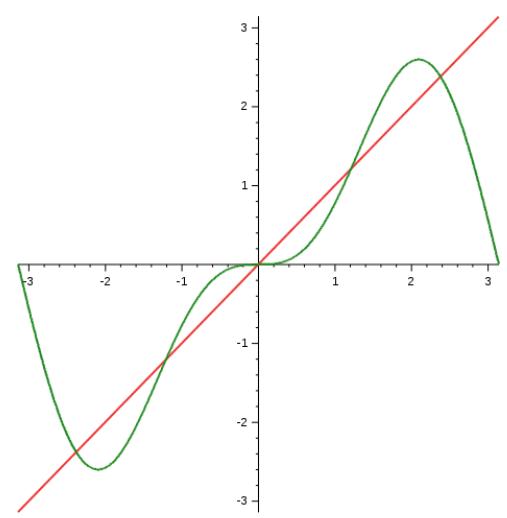

FiguRE 1. $f(x)$ vs $S_{2}(f)(x)$

Fourier series are useful approximations for functions because, like Taylor series, they are infinitely differentiable and easy to (formally) differentiate and integrate. In some cases, Fourier series are in fact much more useful than Taylor series. For example, an infinite Taylor series approximating a function $f$ must be centered at a certain point and may only converge for $x$ in a neighborhood of a certain radius around that point. Fourier series, on the other hand, need not be centered at any specific point. Furthermore, a function must be differentiable to have a (meaningful) Taylor series, whereas a function must be merely integrable to have a Fourier series. If we are to use Fourier series, then we must answer some questions about their convergence because a divergent Fourier series will be of no use when approximating a function. To better study convergence we will examine convolutions, which may be thought of as continuous weighted averages of sorts.

Understanding convolution will allow us to view Fourier series in a different way and provide insight about their summability. The section following convolution is focused on convergence or divergence of infinite Fourier series and the conditions necessary to guarantee such convergence. At the end of the paper, we will look at one application of Fourier analysis to a fundamental principle of geometry: the isoperimetric inequality. The reader is expected to have a strong background in calculus as well as some knowledge of analysis.

\section{Convolution and Kernels}

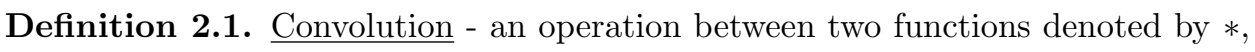
where

$$
(f * g)(x) \equiv \int f(y) g(x-y) d y .
$$


Four our purposes, convolution will be between two $2 \pi$-periodic integrable functions on $\mathbb{R}$ denoted by $*$, where

$$
(f * g)(x) \equiv \frac{1}{2 \pi} \int_{-\pi}^{\pi} f(y) g(x-y) d y .
$$

Remark 2.2. Properties of the convolution operation, which can be proven for continuous functions using the definition of convolution, include:

- $f *(g+h)=(f * g)+(f * h)$

- $(c f) * g=c(f * g)=f *(c g) \forall c \in \mathbb{C}$

- $f * g=g * f$

- $(f * g) * h=f *(g * h)$

- $f * g$ is continuous

- $\widehat{f * g}(n)=\hat{f}(n) \hat{g}(n)$

In fact, all of these properties except the one about continuity hold if either $f$ or $g$ (or both) is merely integrable. A later lemma will even make the continuity property hold if either $f$ or $g$ is continuous and the other is integrable.

Now, let's look at some applications of convolution to Fourier series. We can start with the Dirichlet kernel $D_{n}(x)$, which, when convoluted with a function $f(x)$, yields the $n^{\text {th }}$ partial sum of the Fourier series for $f$. So another way to think about a Fourier series is a convolution with the Dirichlet kernel.

Definition 2.3. Dirichlet kernel - the kernel $D_{N}(x)$ defined as $D_{N}(x)=\sum_{n=-N}^{N} e^{i n x}$.

It is not obvious that this expression is the one satisfying the equation $S_{N}(f)(x)=$ $\left(f * D_{N}\right)(x)$. The following argument shows how it is derived. We know that

$$
S_{N}(f)(x)=\sum_{n=-N}^{N} \hat{f}(n) e^{i n x},
$$

where

$$
\hat{f}(n)=\frac{1}{2 \pi} \int_{-\pi}^{\pi} f(y) e^{-i n y} d y .
$$

We substitute this expression for $\hat{f}$ and put every $e^{i n x}$ inside the integral to get

$$
\frac{1}{2 \pi} \sum_{n=-N}^{N} \int_{-\pi}^{\pi} f(y) e^{i n(x-y)} d y .
$$

Now we can switch the integral and summation signs because everything converges absolutely (this is after all a finite sum) and, factoring out $f(y)$ from the sum, we get

$$
S_{N}(f)(x)=\frac{1}{2 \pi} \int_{-\pi}^{\pi} f(y) \sum_{n=-N}^{N} e^{i n(x-y)} d y .
$$

This fits our definition of convolution and shows that $D_{N}(x)=\sum_{n=-N}^{N} e^{i n x}$.

Remark 2.4. There is a closed-form expression of the Dirichlet kernel that can be derived from the general expression for geometric series $\sum_{n=0}^{N} w^{n}=\frac{1-w^{N+1}}{1-w}$. Splitting the Dirichlet kernel into two sums, $\sum_{n=0}^{N} e^{i n x}$ and $\sum_{n=0}^{N-1} e^{-i(n+1) x}$, and 
applying the series identity and trigonometric identities gives that the $N^{\text {th }}$ Dirichlet kernel is

$$
\frac{\sin \left(\left(N+\frac{1}{2}\right) x\right)}{\sin \left(\frac{x}{2}\right)} .
$$

This is not obviously defined at $x=0$, but a quick look at the summation form of the Dirichlet kernel shows that $D_{N}(0)=2 N+1$, which is also the limit as $x$ goes to 0 of the closed-form Dirichlet kernel.

Now we can introduce the concept of "good kernels," which will aid us in determining the usefulness of a given function $f$ 's Fourier series.

Definition 2.5. A family of good kernels is a sequence of functions $\left\{K_{n}(x)\right\}_{n=1}^{\infty}$ that satisfies the following properties:

- For all $n \geq 1, \quad \frac{1}{2 \pi} \int_{-\pi}^{\pi} K_{n}(x) d x=1$

- There exists $M \geq 0$ such that for all $n \geq 1, \int_{-\pi}^{\pi}\left|K_{n}(x)\right| d x \leq M$

- For every $\delta>0, \int_{\delta \leq|x| \leq \pi}\left|K_{n}(x)\right| d x \rightarrow 0$ as $n \rightarrow \infty$.

Note that if $K_{n}$ is positive, which will often be the case for our purposes, then the first condition implies the second.

Theorem 2.6. Let $\left\{K_{n}(x)\right\}_{n=1}^{\infty}$ be a family of good kernels, and $f$ be an integrable, $2 \pi$-periodic function. Then

$$
\lim _{n \rightarrow \infty}\left(f * K_{n}\right)(x)=f(x)
$$

whenever $f$ is continuous at $x$. If $f$ is continuous everywhere, then the above limit is uniform.

Proof. $f$ is continuous at $x$, so for all $\epsilon^{\prime}>0$, there exists $\delta>0$ such that $|y|<\delta$ implies $|f(x-y)-f(x)|<\epsilon^{\prime}$.

Because good kernels integrate to $2 \pi$ (this is the first property in definition 2.5) we can write

$$
\begin{gathered}
\left(f * K_{n}\right)(x)-f(x)=\frac{1}{2 \pi} \int_{-\pi}^{\pi} K_{n}(y) f(x-y) d y-f(x) \\
=\frac{1}{2 \pi} \int_{-\pi}^{\pi} K_{n}(y)[f(x-y)-f(x)] d y .
\end{gathered}
$$

The triangle inequality then implies

$$
\begin{gathered}
\left|\left(f * K_{n}\right)(x)-f(x)\right| \leq \frac{1}{2 \pi} \int_{-\pi}^{\pi}\left|K_{n}(y)\right||f(x-y)-f(x)| d y= \\
\frac{1}{2 \pi} \int_{|y|<\delta}\left|K_{n}(y)\right||f(x-y)-f(x)| d y+\frac{1}{2 \pi} \int_{\delta \leq|y| \leq \pi}\left|K_{n}(y)\right||f(x-y)-f(x)| d y,
\end{gathered}
$$

where the last equality is obtained by breaking up the domain of integration into two disjoint parts.

By the continuity of $f$ at $x$, the first integral in equation 2.7 is bounded by $\frac{\epsilon^{\prime}}{2 \pi} \int_{|y|<\delta}\left|K_{n}(y)\right| d y$. Because $\int_{-\pi}^{\pi}\left|K_{n}(y)\right| d y \leq M$ (second property in definition $2.5)$, we can write

$$
\frac{\epsilon^{\prime}}{2 \pi} \int_{|y|<\delta}\left|K_{n}(y)\right| d y \leq \frac{\epsilon^{\prime} M}{2 \pi}
$$


To bound the second integral in equation 2.7 we note that $f$ is bounded due to our definition of integrability, say by $B$. Therefore we can use the trivial bound,

$$
\frac{1}{2 \pi} \int_{\delta \leq|y| \leq \pi}\left|K_{n}(y)\right||f(x-y)-f(x)| d y \leq \frac{1}{2 \pi} \int_{\delta \leq|y| \leq \pi}\left|K_{n}(y)\right|(2 B) d y .
$$

Letting $n$ get large, this integral gets arbitrarily small due to the third property in definition 2.5.

Pick $\epsilon^{\prime}>0$ sufficiently small, so that $\frac{M \epsilon^{\prime}}{2 \pi} \leq \epsilon / 2$, and then $n$ sufficiently large (it will depend on $\delta, \epsilon^{\prime}$ ) so that the second integral is bounded by $\epsilon / 2$. Therefore, for these large $n,\left|f * K_{n}(x)-f(x)\right|<\epsilon$, proving the first part of the theorem. As $[-\pi, \pi]$ is compact, any continuous $f$ is uniformly continuous. Thus we can pick $\delta>0$ independently of $x$ and the above analysis shows uniform convergence.

Remark 2.8. The Dirichlet kernel is unfortunately not a good kernel. Using either the closed-form or summation expression, we can see that it does not have the second property of good kernels: $\int_{-\pi}^{\pi} D_{N}(x) d x$ is not bounded for all $N \geq 1$. However, it does satisfy the first property of good kernels, which can be seen intuitively from the closed-form expression: the average value of the Dirichlet kernel is 1 . If the Dirichlet kernel were a good kernel, then by Theorem 2.6, the Fourier series of a function $f(x)$ would converge to $f$ at every point of continuity. This result is not true: several examples of continuous functions whose Fourier series diverge everywhere or almost everywhere have been created by mathematicians like Kolmogoroff, Lebesgue, and Fejér [3].

Despite yielding this dead end, convolution can still be used to prove several important facts about Fourier series. These facts require an understanding of some unconventional types of summation, which can then be used to sum the terms of a Fourier series to the function it hopes to approximate.

Definition 2.9. A series $\sum_{k=0}^{\infty} c_{k}$ is Cesàro summable if the quantity

$$
\sigma_{N}=\frac{S_{0}+S_{1}+\ldots+S_{N-1}}{N}
$$

converges as $\mathrm{N}$ tends to infinity, where the $n^{t h}$ partial sum $S_{n}$ is defined as $\sum_{k=0}^{n} c_{k}$. We say the series is Cesàro summable to $\sigma$ when $\lim _{N \rightarrow \infty} \sigma_{N}=\sigma$. The quantity $\sigma_{N}$ is called the $N^{t h}$ Cesàro mean of the sequence $s_{k}$ or the $N^{t h}$ Cesàro sum of the series $\sum_{k=0}^{\infty} c_{k}$.

Example 2.10. Consider the Grandi series, $1-1+1-1 \ldots$ This series clearly does not converge as $n \rightarrow \infty$. However, using the definition of Cesàro summability, we can see that it is Cesàro summable to $1 / 2$ : We see that $S_{N}=\left\{\begin{array}{ll}1, & N \text { even } \\ 0, & N \text { odd }\end{array}\right.$ and that the sum of the first $N-1$ partial sums is given by $\left\lceil\frac{N+1}{2}\right\rceil$. So

$$
\sigma_{N}=\frac{\lceil(N+1) / 2\rceil}{N}
$$

which converges to $1 / 2$ as $N \rightarrow \infty$ (the first few Cesàro sums are $1,1 / 2,2 / 3,2 / 4, \ldots$ )

Definition 2.11. Fejér kernel - the family of kernels $\left\{F_{n}(x)\right\}_{n=1}^{\infty}$ given by $F_{N}(x)=$ $\frac{D_{0}(x)+\cdots+D_{N-1}(x)}{N}$. 
Remark 2.12. The Fejér kernel convoluted with a function $f$ yields the $N^{\text {th }}$ Cesàro sum of $f$ 's Fourier series. This is not obvious, but it becomes clear considering the following argument. By definition, the $N^{t h}$ Cesàro sum of a Fourier series $\sigma_{N}(f)(x)$ equals

$$
\frac{S_{0}(f)(x)+\cdots+S_{N-1}(f)(x)}{N}=\frac{\left(f * D_{0}\right)(x)+\cdots+\left(f * D_{N-1}\right)(x)}{N},
$$

so by the properties of convolution, the Fejér kernel must be

$$
F_{N}(x)=\frac{D_{0}(x)+\cdots+D_{N-1}(x)}{N} .
$$

Lemma 2.13. $F_{N}(x)=\frac{1}{N} \frac{\sin ^{2}(N x / 2)}{\sin ^{2}(x / 2)}$, and the Fejér kernel is a good kernel.

Proof. First we will establish the closed-form expression for $F_{N}(x)$. Note that, again, it is not well-defined at 0 , but the same argument that we used with the Dirichlet kernel applies here. We know $D_{N}(x)=\frac{\sin ((N+1 / 2) x)}{\sin (x / 2)}$, so

$$
\begin{gathered}
N F_{N}=\sum_{n=0}^{N-1} D_{N}(x)=\frac{\sin (x / 2)}{\sin (x / 2)}+\cdots+\frac{\sin ((N-1 / 2) x)}{\sin (x / 2)} . \\
=\frac{\sin (x / 2) \sin (x / 2)+\sin (3 x / 2) \sin (x / 2)+\cdots+\sin ((N-1 / 2)(x)) \sin (x / 2)}{\sin ^{2}(x / 2)}
\end{gathered}
$$

Now we can apply the identity

$$
\sin ((m+1 / 2) x) \sin (x / 2)=\frac{1}{2}(\cos (m x)-\cos ((m+1) x)),
$$

which causes quite a bit of cancellation and leaves us with

$$
=\frac{1 / 2(1-\cos (N x))}{\sin ^{2}(x / 2)} .
$$

Applying the previous identity to $\sin (N x / 2) \sin (N x / 2)$,

$$
=\frac{\sin ^{2}(N x / 2)}{\sin ^{2}(x / 2)} \text {. }
$$

Dividing by $\mathrm{N}$ then yields $F_{N}=\frac{\sin ^{2}(N x / 2)}{N \sin ^{2}(x / 2)}$. Now, $F_{N}$ clearly has the first property of good kernels because $D_{N}$ does. Notice that $F_{N}$ is strictly positive, so the first property of good kernels implies the second. Finally, for all $\epsilon>0$ there exists $\delta$ such that $\delta \leq|x| \leq \pi$ implies $\sin ^{2}(x / 2) \geq \epsilon>0$, so $F_{N}(x) \leq \frac{1}{N \epsilon}$, which implies that

as $N \rightarrow \infty$.

$$
\int_{\delta \leq|x| \leq \pi}\left|F_{N}(x)\right| d x \rightarrow 0
$$

Applying Theorem 2.6 to Fejér kernels gives us an important theorem about Cesàro summability of Fourier series.

Theorem 2.14. If $f$ is integrable and $2 \pi$-periodic, then the Fourier series of $f$ is Cesàro summable to $f$ at every point of continuity of $f$. Moreover, if $f$ is continuous, then the Fourier series of $f$ is uniformly Cesàro summable to $f$.

There is also a Corollary that will be needed later and is an immediate consequence of this Theorem: 
Corollary 2.15. Functions that are continuous on the interval $[-\pi, \pi]$ with $f(-\pi)=$ $f(\pi)$ can be uniformly approximated by trigonometric polynomials.

Proof. The partial sums of f's Fourier series used in the Cesàro sums, and thus the Cesàro sums themselves, are trigonometric polynomials that uniformly sum to $f$ as $N \rightarrow \infty$.

This method lets us use a function's Fourier series to approximate it, albeit via an unusual type of summation. Let's look at a different type that can be used to sum the terms of a Fourier series.

Definition 2.16. A complex series $\sum_{k=0}^{\infty} c_{k}$ is Abel summable if, for all $r$ in [0,1), the series $\sum_{k=0}^{\infty} c_{k} r^{k}=A(r)$ converges and $\lim _{r \rightarrow 1} A(r)$ exists. The quantities $A(r)$ are Abel means, and we say the series is Abel summable to $s$ if $\lim _{r \rightarrow 1} A(r)=s$.

Example 2.17. This will serve as an example for both an Abel-summable series and the fact that not every Abel-summable series is also Cesàro-summable (although in fact, every Cesàro-summable series is Abel-summable). Consider the series $1-4+9-16 \ldots+(-1)^{k}(k+1)^{2}$. Letting $S_{0}=1$ and attempting a Cesàro sum, we see that

$$
\sigma_{N}=\frac{\sum_{k=0}^{N-1} S_{k}}{N}
$$

where $S_{k}$ is the $k^{t h}$ partial sum of the series. Examining the sequence of partial sums, we see that $S_{k}=(-1)^{k} \frac{(k+1)(k+2)}{2}$, so the Cesàro sum becomes

$$
\sigma_{N}=\frac{\sum_{k=0}^{N-1}(-1)^{k} \frac{(k+1)(k+2)}{2}}{N} .
$$

This sum diverges as $N \rightarrow \infty$ by the Limit Test - the terms do not go to 0 . Thus the series is not Cesàro summable. Attempting to Abel sum, on the other hand, yields

$$
A(r)=\sum_{k=0}^{\infty}(-1)^{k}(k+1)^{2} r^{k}
$$

which is the power series for $\frac{1-r}{(1+r)^{3}}$ centered at 0 (or for $|r|<1$ ). Thus the limit as $r \rightarrow 1$ is 0 , so the series is Abel summable to 0 (note that $A(1) \neq 0$, but the limit is 0$)$.

Definition 2.18. Poisson kernel-the family of kernels $\left\{P_{r}(x)\right\}, 0 \leq r<1$ given by $P_{r}(x)=\sum_{n=-\infty}^{\infty} r^{|n|} e^{i n x}$.

Remark 2.19. The Poisson kernel is important because it gives us the Abel sums of a function's Fourier series: $A_{r}(f)(x)=\left(f * P_{r}\right)(x)$. To see this, we need only rearrange some terms to fit our definition of convolution:

$$
\begin{gathered}
A_{r}(f)(x)=\sum_{n=-\infty}^{\infty} r^{|n|} \hat{f}(n) e^{i n x}=\sum_{n=-\infty}^{\infty} r^{|n|}\left(\frac{1}{2 \pi} \int_{-\pi}^{\pi} f(y) e^{-i n y} d y\right) e^{i n x} \\
=\frac{1}{2 \pi} \int_{-\pi}^{\pi} f(y)\left(\sum_{n=-\infty}^{\infty} r^{|n|} e^{i n(x-y)}\right) d y
\end{gathered}
$$

Switching the integral and infinite sum sign is justified due to the uniform convergence of the series. This then yields that $P_{r}(x)=\sum_{n=-\infty}^{\infty} r^{|n|} e^{i n x}$. 
Remark 2.20. A closed-form expression for the Poisson kernel is $\frac{1-r^{2}}{1-2 r \cos x+r^{2}}$. This is derived by splitting $P_{r}(x)$ into

$$
\sum_{n=0}^{\infty}\left(r e^{i x}\right)^{n}+\sum_{n=1}^{\infty}\left(r e^{-i x}\right)^{n}
$$

and applying identities for geometric series and Euler's formula. Defining this expression at $x=0$ requires a procedure similar to defining the Dirichlet and Fejér kernels at this point. It should also be noted that this expression holds only for $0 \leq r<1$.

Lemma 2.21. For any sequence $a_{n} \in[0,1)$ such that $\lim a_{n}=1, P_{a_{n}}$ is a family of good kernels.

Proof. If $1 / 2 \leq r<1$ and $\delta \leq|x| \leq \pi$, then the denominator $1-2 r \cos x+r^{2} \geq$ $c_{\delta}>0$, giving us a constant positive lower bound.

Thus $P_{r}(x) \leq \frac{(1-r)^{2}}{c_{\delta}}$ when $\delta \leq|x| \leq \pi$, and this upper bound on the Poisson kernel becomes arbitrarily small when $r$ tends to 1 from below, so for every $\delta>0$, the integral of the Poisson kernel goes to 0 as r goes to 1: $\int_{y \geq 0} P_{r}(x) \rightarrow 0$ as $r \rightarrow 1$. This is the definition of the third property of good kernels.

The closed-form expression shows that $P_{r}(x)$ is always positive when $0 \leq r<1$, so the first property would imply the second. For the first property, integrating the Poisson kernel in summation form yields only $\frac{1}{2 \pi} \int_{-\pi}^{\pi} e^{i(0) x} d x$ - all terms where $n \neq 0$ integrate to 0 . This expression equals 1 , so all three properties of good kernels are satisfied.

Applying Theorem 2.6 to this lemma yields the following theorem.

Theorem 2.22. The Fourier series of an integrable $2 \pi$-periodic function is Abel summable to $f$ at every point of continuity. Moreover, if $f$ is continuous, then the Fourier series of $f$ is uniformly Abel summable to $f$.

This result demonstrates a particular case of the general fact that every Cesàro summable series is Abel summable to the same limit.

\section{Criteria for Convergence}

Now we will move away from considering unusual methods of summation in order to guarantee convergence and start to consider convergence of Fourier series summed in the usual way. The reader should assume in this section that any function $f$ considered is $2 \pi$-periodic (most proofs can be extended to any periodic function). In order to do move forward, we need to understand the vector space of complex-valued integrable functions on $[0,2 \pi]$. This is a vector space over $\mathbb{C}$. We define an inner product as

$$
(f, g)=\frac{1}{2 \pi} \int_{0}^{2 \pi} f(x) \overline{g(x)} d x .
$$

This inner product is bilinear, and the norm of $f$ or $\|f\|$ is

$$
\left(\frac{1}{2 \pi} \int_{0}^{2 \pi}|f(x)|^{2} d x\right)^{1 / 2}
$$


Let $e_{n}(x)=e^{i n x}$. It is important for the reader to observe that under this inner product, the family $\left\{e_{n}\right\}_{n \in \mathbb{Z}}$ is orthonormal, or

$$
\left(e_{n}, e_{m}\right)= \begin{cases}1 & \text { if } n=m \\ 0 & \text { if } n \neq m\end{cases}
$$

Notice also that

$$
\left(f, e_{n}\right)=\frac{1}{2 \pi} \int_{0}^{2 \pi} f(x) e^{-i n x} d x=a_{n} .
$$

Recall that $S_{N}(f)=\sum_{|n| \leq N} a_{n} e_{n}$, which is a sum of these inner products with coefficients $e_{n}$. Now we can introduce a small lemma:

\section{Lemma 3.1.}

$$
\left(f-\sum_{|n| \leq N} a_{n} e_{n}\right) \perp \sum_{|n| \leq N} b_{n} e_{n}
$$

for any complex numbers $b_{n}$ under the inner product previously defined.

Proof. Applying the properties of the inner product,

$$
\left(f-\sum_{|n| \leq N} a_{n} e_{n}, \sum_{|n| \leq N} b_{n} e_{n}\right)=\left(f, \sum_{|n| \leq N} b_{n} e_{n}\right)-\left(\sum_{|n| \leq N} a_{n} e_{n}, \sum_{|n| \leq N} b_{n} e_{n}\right)
$$

Applying the orthonormal property of $\left\{e_{n}\right\}$, bilinearity, and the fact that $\left(f, e_{n}\right)=$ $a_{n}$,

$$
=\left(a_{0} b_{0}+\ldots+a_{n} b_{n}+a_{-n} b_{-n}\right)-\left(a_{0} b_{0}+\ldots+a_{n} b_{n}+a_{-n} b_{-n}\right)=0
$$

Intuitively, this lemma says that subtracting all of the $e_{1}, \ldots, e_{n}$ "components" of $f$ leaves a function that is perpendicular to the space spanned by $\left\{e_{1}, \ldots, e_{n}\right\}$.

Now we can apply the Pythagorean Theorem to the decomposition

$$
f=f-\sum_{|n| \leq N} a_{n} e_{n}+\sum_{|n| \leq N} a_{n} e_{n}
$$

where $f-\sum_{|n| \leq N} a_{n} e_{n} \perp \sum_{|n| \leq N} a_{n} e_{n}$ by the previous lemma, to get $\|f\|^{2}=$ $\left\|f-\sum_{|n| \leq N} a_{n} e_{n}\right\|^{2}+\left\|\sum_{|n| \leq N} a_{n} e_{n}\right\|^{2}$. The last sum is equal to

$$
\left(\sum_{|n| \leq N} a_{n} e_{n}, \sum_{|n| \leq N} a_{n} e_{n}\right)
$$

which reduces to

$$
a_{0} \overline{a_{0}}\left(e_{0}, e_{0}\right)+\ldots+a_{n} \overline{a_{n}}\left(e_{n}, e_{n}\right)+a_{-n} \overline{a_{-n}}\left(e_{-n}, e_{-n}\right)=\sum_{|n| \leq N}\left|a_{n}\right|^{2} .
$$

Our final result is thus

$$
\|f\|^{2}=\left\|f-S_{N}(f)\right\|^{2}+\sum_{|n| \leq N}\left|a_{n}\right|^{2}
$$

where $S_{N}(f)$ is the $N^{t h}$ partial sum of $f$ 's Fourier series and $a_{n}$ are the Fourier coefficients. From the above we can deduce the Best Approximation Lemma.

Lemma 3.3. Best Approximation Lemma: $\left\|f(x)-S_{N}(f)(x)\right\| \leq \| f(x)-$ $\sum_{|n|=1}^{N} c_{n} e_{n} \|$ 
Proof. We start by rewriting the difference between $f$ and any sum of trigonometric polynomials to include $f$ 's Fourier series:

$$
f-\sum_{|n| \leq N} c_{n} e_{n}=f-S_{N}(f)+\sum_{|n| \leq N}\left(a_{n}-c_{n}\right) e_{n}
$$

Applying the Pythagorean Theorem to the perpendicular components $f-S_{N}(f)$ and $\sum_{|n| \leq N}\left(a_{n}-c_{n}\right) e_{n}$

$$
\left\|f-\sum_{|n| \leq N} c_{n} e_{n}\right\|^{2}=\left\|f-S_{N}(f)\right\|^{2}+\left\|\sum_{|n| \leq N} b_{n} e_{n}\right\|^{2}
$$

where $b_{n}=a_{n}-c_{n}$. This gives us the desired result because $\|g\|^{2}$ is never negative:

$$
\left\|f(x)-S_{N}(f)(x)\right\| \leq\left\|f(x)-\sum_{|n|=1}^{N} c_{n} e_{n}\right\| .
$$

Equality holds when the last norm is 0 (when $a_{n}=c_{n}$ ).

This Lemma shows that the Fourier approximation of a function is the best approximation of the form $\sum_{|n|=1}^{N} c_{n} e_{n}$ where $c_{k} \in \mathbb{C}$. Together with the following lemma about approximating integrable functions, we can prove our first important convergence theorem.

Lemma 3.4. Suppose $f(x)$ is a $2 \pi$-periodic integrable function that is bounded by $B$. Then there exists an infinite sequence $\left\{f_{k}\right\}$ of continuous, $2 \pi$-periodic functions, $k=1,2, \ldots$ such that

$$
\sup _{x \in[-\pi, \pi]}\left|f_{k}(x)\right| \leq B, \quad \forall k=1,2, \ldots
$$

and

$$
\int_{-\pi}^{\pi}\left|f(x)-f_{k}(x)\right| d x \rightarrow 0
$$

as $k \rightarrow \infty$.

Proof. First we assume $f$ is real (in general, apply the following proof to the real and imaginary parts of a complex-valued function separately). Given $\epsilon>0$, we can choose a partition $-\pi=x_{0}<x_{1}<\cdots<x_{N}=\pi$ of the interval $[-\pi, \pi]$ so that the upper and lower sums of $f$ differ by at most $\epsilon$ because $f$ is integrable. Now we denote $f^{*}$ the step function defined by

$$
f^{*}(x)=\sup _{x_{j-1} \leq y \leq x_{j}} f(y) \text { if } x \in\left[x_{j-1}, x_{j}\right)
$$

for $1 \leq j \leq N$. Now $f^{*}$ is obviously bounded by $B$, and moreover

$$
\int_{-\pi}^{\pi}\left|f^{*}(x)-f(x)\right| d x=\int_{-\pi}^{\pi}\left(f^{*}(x)-f(x)\right) d x<\epsilon
$$

This function $f^{*}$ is not continuous, but we can modify it to make it so. For small $\delta>0$, let $\tilde{f}(x)=f^{*}(x)$ when the distance between $x$ and any partition point $x_{0} \ldots x_{N}$ is at least $\delta$. When $x$ is within $\delta$ of a partition point, define $\tilde{f}(x)$ to be the linear function connecting $f^{*}(x-\delta)$ and $f^{*}(x+\delta)$. This makes $\tilde{f}(x)$ continuous - it helps to visualize $f^{*}(x)$ as a step function with one step above each interval $\left[x_{j-1}, x j\right)$. Then think of $\tilde{f}$ as $f^{*}$ with $\delta$ removed from each end of every step and 
lines drawn connecting the new ends of all of the steps.

Now let $\tilde{f}=0$ in the neighborhood of $x=-\pi$ and $x=\pi$. This lets us extend $\tilde{f}$ to a $2 \pi$-periodic function. The absolute value of this extension is still less than our bound on $f, B$. Moreover, $\tilde{f}$ differs from $f^{*}$ only in the $\mathrm{N}$ intervals of length $2 \delta$ surrounding the partition points. We thus have

$$
\int_{-\pi}^{\pi}\left|f^{*}(x)-\tilde{f}(x)\right| d x \leq 2 B N(2 \delta) .
$$

If we choose sufficiently small $\delta$, we then get

$$
\int_{-\pi}^{\pi}\left|f^{*}(x)-\tilde{f}(x)\right| d x<\epsilon
$$

Combining this equation and 3.5 , the triangle inequality yields

$$
\int_{-\pi}^{\pi}|f(x)-\tilde{f}(x)| d x<2 \epsilon .
$$

Denoting by $f_{k}$ our function $\tilde{f}$ so constructed, when $2 \epsilon=1 / k$, we see that the sequence $\left\{f_{k}\right\}$ has the properties required by the lemma. Letting $k \rightarrow \infty$ is analogous to letting $\delta \rightarrow 0$ and $N \rightarrow \infty$.

Now we get to the theorem.

Theorem 3.6. Mean Square Convergence-Suppose $f$ is integrable on the interval $[0,2 \pi]$. Then

$$
\frac{1}{2 \pi} \int_{0}^{2 \pi}\left|f(x)-S_{N}(f)(x)\right|^{2} d x \rightarrow 0
$$

as $N \rightarrow \infty$.

Proof. Remember that $\left\|f-S_{N}(f)\right\|=\left(\frac{1}{2 \pi} \int_{0}^{2 \pi}\left|f(x)-S_{N}(f)\right|^{2} d x\right)^{1 / 2}$. A restatement of Corollary 2.15 is that if $f$ is continuous on the interval $[0,2 \pi]$ and $2 \pi$ periodic, then given $\epsilon>0$, there exists a trigonometric polynomial $P$ such that

$$
|f(x)-P(x)|<\epsilon
$$

for all $x$. Let the degree of $P$ be $M$. This means that $\left(P, e_{n}\right) \neq 0$ implies $n \leq M$. Squaring and integrating both sides of the inequality yields, by our definition of the norm of $f$ and the Best Approximation Lemma,

$$
\left\|f-S_{N}(f)\right\|<\epsilon
$$

whenever $N \geq M$. This gives the desired result when $f$ is continuous. When $f$ is merely integrable, we can apply Lemma 3.4 to give us a continuous function $g$ that satisfies

and

$$
\sup _{x \in[-\pi, \pi]}|g(x)| \leq \sup _{x \in[-\pi, \pi]}|f(x)|=B
$$

$$
\int_{-\pi}^{\pi}|f(x)-g(x)| d x<\epsilon^{2}
$$

Using our definition of norm and the fact that $\sup _{x \in[-\pi, \pi]}|f(x)|=B$,

$$
\|f-g\|^{2}=\frac{1}{2 \pi} \int_{0}^{2 \pi}|f(x)-g(x)|^{2} d x
$$




$$
\begin{gathered}
=\frac{1}{2 \pi} \int_{0}^{2 \pi}|f(x)-g(x)||f(x)-g(x)| d x \\
\leq \frac{2 B}{2 \pi} \int_{0}^{2 \pi}|f(x)-g(x)| d x
\end{gathered}
$$

which, by Lemma 3.4,

$$
\leq C \epsilon^{2}
$$

It should be noted here that changing the limits of integration has no effect because the functions are $2 \pi$-periodic. Now we know that there exists a trigonometric polynomial $P$ such that $\|g-P\|<\epsilon$ because $g$ is continuous. Then $\|f-P\|<C^{\prime} \epsilon$. We know by the Best Approximation Lemma that $P$ is the Fourier series of $f$, giving us the desired result.

This Theorem, together with equation 3.2, gives us Parseval's identity.

Theorem 3.7. Parseval's identity: $\sum_{n=-\infty}^{\infty}\left|a_{n}\right|^{2}=\|f\|^{2}$

Proof. Recall equation 3.2

$$
\|f\|^{2}=\left\|f-S_{N}(f)\right\|^{2}+\sum_{|n| \leq N}\left|a_{n}\right|^{2} .
$$

We just proved that the first quantity on the right goes to 0 as $n \rightarrow \infty$, so the equation considered at $n=\infty$ gives us the desired result.

In fact, we can prove a more general version of Parseval's Identity.

Lemma 3.8. Suppose $F$ and $G$ are integrable and $2 \pi$-periodic with $F \sim \sum a_{n} e^{i n x}$ and $G \sim \sum b_{n} e^{i n x}$. Then

$$
(F, G)=\frac{1}{2 \pi} \int_{0}^{2 \pi} F(x) \overline{G(x)} d x=\sum_{n=-\infty}^{\infty} a_{n} \overline{b_{n}} .
$$

Proof. This proof uses some messy but easy algebra to rewrite $(F, G)$.

$$
\begin{gathered}
(F, G)=\frac{1}{4}((F+G, F+G)-(F-G, F-G)+i(F+i G, F+i G)-i(F-i G, F-i G)) \\
=\frac{1}{4}\left(\|F+G\|^{2}-\|F-G\|^{2}+i\|F+i G\|^{2}-i\|F-i G\|^{2}\right)
\end{gathered}
$$

By Parseval's Identity and more algebra,

$$
=\sum_{n=-\infty}^{\infty} a_{n} \overline{b_{n}}
$$

Mean-square convergence and Parseval's identity tell us some important things about convergence of expressions closely related to Fourier series. As for Fourier series themselves, the following few theorems give conditions that guarantee convergence of a function $f$ 's Fourier series to $f$. We can start with a theorem that will make later proofs easier.

Theorem 3.9. Let $f$ be a continuous function with associated Fourier coefficients $\left\{a_{n}\right\}$. Then if $\sum_{n=-\infty}^{\infty}\left|a_{n}\right|<\infty$, we have $S_{N}(f) \rightarrow f$ uniformly as $N \rightarrow \infty$. 
Proof. Let $g(x)=\sum_{n=-\infty}^{\infty} a_{n} e_{n}$ and $S_{N}(f)(x)=\sum_{|n| \leq N} a_{n} e_{n}$. Note that $g(x)$ is defined everywhere because $\sum_{n=-\infty}^{\infty}\left|a_{n}\right|<\infty$, which is a consequence of our conditions. First we will prove that

$$
\lim _{n \rightarrow \infty} \sum_{|n| \leq N} a_{n} e_{n}=g(x)
$$

and that this limit is uniform. By the definitions of $S_{N}$ and $g(x)$ and the triangle inequality, we have

$$
\left|S_{N}(f)(x)-g(x)\right|=\left|\sum_{|n|>N} a_{n} e_{n}\right| \leq \sum_{|n|>N}\left|a_{n}\right| .
$$

This last quantity can be made arbitrarily small with large $N$ because $\sum_{n=-\infty}^{\infty}\left|a_{n}\right|<$ $\infty$, proving our first claim. Now we know that $g(x)$ is the uniform limit of continuous, $2 \pi$-periodic functions, so $g(x)$ is also continuous and $2 \pi$-periodic. Now note that for large $N$ (such that $N>|n|)$,

$$
\left(S_{N}(f)(x), e_{n}\right)=\frac{1}{2 \pi} \int_{0}^{2 \pi}\left(\sum_{|n| \leq N} a_{n} e^{i n x}\right) e^{-i n x} d x=a_{n} .
$$

This, along with the fact that $S_{N}(f)(x) \rightarrow g(x)$ uniformly as $N \rightarrow \infty$, tells us that $\left(g(x), e_{n}\right)=a_{n}$. In other words, $g(x)$ 's Fourier series is identical to $f(x)$ 's. Now we can consider the function $f-g$. Because $f$ and $g$ have identical Fourier coefficients, this function's Fourier coefficients are all 0 by distributivity of the integral. Finally, we apply Parseval's Identity to this new function:

$$
0=\sum_{n=-\infty}^{\infty}\left|a_{n}\right|^{2}=\|f-g\|^{2}=\frac{1}{2 \pi} \int_{0}^{2 \pi}|(f-g)(x)|^{2} d x
$$

We know that if $\int|(f-g)(x)| d x=0$ for continuous $f$ and $g$, then $f-g=0$ or $f=g$ because any point of inequality would require inequality in a surrounding region and thus a nonzero integral. This gives us the desired result.

Lemma 3.10. Let $f$ be a differentiable function with an integrable derivative. Then the following holds:

$$
\widehat{f}^{\prime}(n)=i n \hat{f}(n) .
$$

In other words, the derivative of $f$ 's Fourier series equals the Fourier series for $f^{\prime}$.

Proof. Starting with the definition,

$$
2 \pi \hat{f}(n)=\int_{0}^{2 \pi} f(x) e^{-i n x} d x .
$$

Applying integration by parts,

$$
=\left[f(x) \frac{-e^{-i n x}}{i n}\right]_{0}^{2 \pi}+\frac{1}{i n} \int_{0}^{2 \pi} f^{\prime}(x) e^{-i n x} d x .
$$

The first term vanishes because $f$ is periodic, leaving

$$
2 \pi \hat{f}(n)=\frac{1}{i n} \int_{0}^{2 \pi} f^{\prime}(x) e^{-i n x} d x .
$$


Some rearranging yields the desired result,

$$
\operatorname{in} \hat{f}(n)=\frac{1}{2 \pi} \int_{0}^{2 \pi} f^{\prime}(x) e^{-i n x} d x=\widehat{f}^{\prime}(n) .
$$

This lemma gives us a tool we will use several times in the rest of this section. Now we can prove our first convergence theorem.

Theorem 3.11. If $f$ is $2 \pi$-periodic and has an integrable derivative, then its Fourier series converges absolutely and uniformly to $f$.

Proof. We will prove that the Fourier series converges and then invoke Theorem 3.9 to prove that it converges to $f$. We start by removing the term $a_{0}$,

$$
\sum_{n=-\infty}^{\infty} a_{n}=a_{0}+\sum_{|n|=1}^{\infty} a_{n} .
$$

Now we just need to prove convergence for the last sum. Using the Cauchy-Schwarz Inequality,

$$
\left(\sum_{|n|=1}^{\infty} a_{n}\right)^{2} \leq \sum_{|n|=1}^{\infty} \frac{1}{n^{2}} \sum_{n=-\infty}^{\infty} n^{2} a_{n}^{2}
$$

The sum $\sum_{|n|=1}^{\infty} \frac{1}{n^{2}}$ equals $\frac{\pi^{2}}{3}$. For the last sum, we have the following by Lemma 3.10 and Parseval's Identity:

$$
\sum_{n=-\infty}^{\infty}\left|n^{2} a_{n}^{2}\right|=\sum_{n=-\infty}^{\infty}\left|\widehat{f}^{\prime}(n)\right|^{2}=\left\|f^{\prime}\right\|^{2}<\infty
$$

where the norm exists because $f$ has an integrable derivative, giving us the desired result.

This theorem will do for continuously differentiable functions, but what can we say about functions that aren't continuously differentiable? The following few theorems are about functions with less restrictions.

Lemma 3.12. For any $2 \pi$-periodic and integrable function $f$,

$$
\hat{f}(n)=-\frac{1}{2 \pi} \int_{-\pi}^{\pi} f(x+\pi / n) e^{-i n x} d x
$$

and hence

$$
\hat{f}(n)=\frac{1}{4 \pi} \int_{-\pi}^{\pi}[f(x)-f(x+\pi / n)] e^{-i n x} d x
$$

Proof. We have by definition

$$
\hat{f}(n)=\frac{1}{2 \pi} \int_{-\pi}^{\pi} f(y) e^{-i n y} d y .
$$

Notice that we can make substitutions without changing the bounds of integration as long as we are integrating over the period of $f$ because $f$ is periodic. Making the substitution $y=x+\pi / n$, we have

$$
\hat{f}(n)=\frac{1}{2 \pi} \int_{-\pi}^{\pi} f(x+\pi / n) e^{-i n(x+\pi / n)} d x
$$




$$
=\frac{1}{2 \pi} \int_{-\pi}^{\pi} f(x+\pi / n) e^{-i n x} e^{-\pi i} d x
$$

Applying a form of Euler's Identity,

$$
=-\frac{1}{2 \pi} \int_{-\pi}^{\pi} f(x+\pi / n) e^{-i n x} d x,
$$

giving us the first desired result. Then we see that

so

$$
2 \hat{f}(n)=\frac{1}{2 \pi}\left(\int_{-\pi}^{\pi} f(x) e^{-i n x} d x-\int_{-\pi}^{\pi} f(x+\pi / n) e^{-i n x} d x\right)
$$

$$
\hat{f}(n)=\frac{1}{4 \pi} \int_{-\pi}^{\pi}[f(x)-f(x+\pi / n)] e^{-i n x} d x,
$$

giving us the second part of the lemma.

Now we will introduce a quick definition to make our next theorem more clear.

Definition 3.13. A function satisfies a Hölder condition of order $\alpha$ if

$$
|f(x+h)-f(x)| \leq C|h|^{\alpha}
$$

for some $0<\alpha \leq 1$, some $C>0$, and all $x, h$.

Theorem 3.14. If $f$ is integrable and $2 \pi$-periodic and satisfies a Hölder condition of order $\alpha$, namely

$$
|f(x+h)-f(x)| \leq C|h|^{\alpha}
$$

for some $0<\alpha \leq 1$, some $C>0$, and all $x, h$, then

$$
\hat{f}(n)=O\left(1 /|n|^{\alpha}\right) .
$$

Proof. Using the previous lemma, we know that

$$
|\hat{f}(n)|=\frac{1}{4 \pi}\left|\int_{-\pi}^{\pi}[f(x)-f(x+\pi / n)] e^{-i n x} d x\right| .
$$

By the triangle inequality for integrals, we see that

$$
|\hat{f}(n)| \leq \frac{1}{4 \pi} \int_{-\pi}^{\pi}|f(x)-f(x+\pi / n)|\left|e^{-i n x}\right| d x .
$$

Now we can apply the Hölder condition to get

$$
|\hat{f}(n)| \leq \frac{1}{4 \pi} \int_{-\pi}^{\pi} C|\pi / n|^{\alpha}\left|e^{-i n x}\right| d x .
$$

Applying $\int_{-\pi}^{\pi}\left|e^{-i n x}\right| d x=2 \pi$ yields

$$
|\hat{f}(n)| \leq \frac{C \pi^{\alpha}}{2|n|^{\alpha}}
$$

which is the desired result.

One might be tempted to try to use this theorem to prove convergence of a function $f$ 's Fourier series whenever $f$ satisfies some sort of Hölder condition. Together with Parseval's Identity, this theorem tells us that the Fourier series of a function $f$ converges absolutely and uniformly to $f$ at every point of continuity as long as $f$ satisfies a Hölder condition of order $\alpha$ and the series $\sum_{n=1}^{\infty} \frac{1}{n^{\alpha}}$ converges. Unfortunately, this sum diverges for all $\alpha$ such that $0<\alpha \leq 1$. Thus the theorem gives a lower bound on the speed of the decay of the Fourier coefficients, but this bound is 
unfortunately too low to guarantee convergence. An approach similar to Theorem 3.11 may seem logical, but this will lead us nowhere. Instead, we will use a different method of proof to guarantee convergence given certain Hölder conditions.

For the following sequence of lemmas and the resulting proof, let $f$ be a $2 \pi$ periodic function which satisfies a Hölder condition of constants $K$ and $\alpha$, or

$$
|f(x)-f(y)| \leq K|x-y|^{\alpha} .
$$

Lemma 3.15. Let $g_{h}(x)=f(x+h)-f(x-h)$ for every positive $h$. Then

$$
\frac{1}{2 \pi} \int_{0}^{2 \pi}\left|g_{h}(x)\right|^{2} d x=\sum_{n=-\infty}^{\infty} 4|\sin (n h)|^{2}|\hat{f}(n)|^{2}
$$

and

$$
\sum_{n=-\infty}^{\infty}|\sin (n h)|^{2}|\hat{f}(n)|^{2} \leq \frac{K^{2} h^{2 \alpha} 2^{2 \alpha}}{4}
$$

Proof. We will prove each part of the lemma separately. For the first part, we will start by proving $\widehat{g_{h}}(n)=2 i \sin (n h) \hat{f}(n)$. Using the definition of $\widehat{g_{h}}(n)$,

$$
\begin{gathered}
\widehat{g_{h}}(n)=\frac{1}{2 \pi} \int_{-\pi}^{\pi}(f(x+h)-f(x-h)) e^{-i n x} d x \\
=\frac{1}{2 \pi}\left(\int_{-\pi}^{\pi} f(x+h) e^{-i n x} d x-\int_{-\pi}^{\pi} f(x-h) e^{-i n x} d x\right)
\end{gathered}
$$

Again note that we can change variables without changing the bounds of integration. Using the changes of variables $y=x+h$ and $z=x-h$,

$$
\begin{gathered}
=\frac{1}{2 \pi}\left(\int_{-\pi}^{\pi} f(y) e^{-i n y} e^{i n h} d x-\int_{-\pi}^{\pi} f(z) e^{-i n z} e^{-i n h} d x\right) \\
=e^{i n h} \hat{f}(n)-e^{-i n h} \hat{f}(n)
\end{gathered}
$$

This, after applying Euler's Identity, equals $2 i \sin (n h) \hat{f}(n)$, proving the equation at the beginning of the proof. Applying Parseval's Identity to this equation yields the first desired result,

$$
\frac{1}{2 \pi} \int_{0}^{2 \pi}\left|g_{h}(x)\right|^{2} d x=\sum_{n=-\infty}^{\infty} 4|\sin (n h)|^{2}|\hat{f}(n)|^{2} .
$$

For the second part of the lemma, we use the fact that, by our assumptions, $\left|g_{h}(x)\right| \leq K(2 h)^{\alpha}$. Applying our result from the first part of the proof,

$$
\sum_{n=-\infty}^{\infty} 4|\sin (n h)|^{2}|\hat{f}(n)|^{2} \leq \frac{1}{2 \pi} \int_{0}^{2 \pi}\left|K(2 h)^{\alpha}\right|^{2} d x=K^{2} 2^{2 \alpha} h^{2 \alpha} .
$$

Dividing by 4 yields the upper bound $\frac{K^{2} 2^{2 \alpha} h^{2 \alpha}}{4}$, the desired result.

The next lemma applies the previous one and brings us closer to our goal of some sort of Hölder condition as the sole requirement for Fourier convergence.

Lemma 3.16. Let $p$ be a positive integer. Then $\sum_{2^{p-1}<|n| \leq 2^{p}}|\hat{f}(n)|^{2} \leq \frac{K^{2} \pi^{2 \alpha}}{2^{2 \alpha p+1}}$. 
Proof. This proof is based on the idea of dyadic shells, a type of argument that appears often in Fourier analysis. The intuition behind this type of argument is that regularity at scale $t$ translates to summability at scale $1 / t$. We start by letting $h$ in the previous lemma equal $\pi / 2^{p+1}$. This yields

$$
\sum_{2^{p-1}<|n| \leq 2^{p}}\left|\sin \left(\frac{n \pi}{2^{p+1}}\right)\right|^{2}|\hat{f}(n)|^{2} \leq \frac{K^{2} 2^{2 \alpha} \pi^{2 \alpha}}{2^{2+2 \alpha p+2 \alpha}}=\frac{K^{2} \pi^{2 \alpha}}{2^{2 \alpha p+2}} .
$$

Now because we are only summing over $2^{p-1}<|n| \leq 2^{p}$, the argument of $\sin$ is between $\frac{\pi}{4}$ and $\frac{\pi}{2}$, and we have

$$
\frac{1}{2} \sum_{2^{p-1}<|n| \leq 2^{p}}|\hat{f}(n)|^{2}<\sum_{2^{p-1}<|n| \leq 2^{p}}\left|\sin \left(\frac{n \pi}{2^{p+1}}\right)\right|^{2}|\hat{f}(n)|^{2} .
$$

Connecting the inequalities and rearranging yields the desired result:

$$
\sum_{2^{p-1}<|n| \leq 2^{p}}|\hat{f}(n)|^{2} \leq \frac{K^{2} \pi^{2 \alpha}}{2^{2 \alpha p+1}} .
$$

Now we come to a strong theorem about Fourier convergence.

Theorem 3.17. Bernstein's Theorem: If $f$ is continuous and satisfies a Hölder condition of order $\alpha>1 / 2$, then the Fourier series of $f$ converges absolutely to $f$.

Proof. Using the Cauchy-Schwarz Inequality,

$$
\left(\sum_{2^{p-1}<|n| \leq 2^{p}}|\hat{f}(n)|\right)^{2} \leq \sum_{2^{p-1}<|n| \leq 2^{p}}|\hat{f}(n)|^{2} \sum_{2^{p-1}<|n| \leq 2^{p}} 1 .
$$

The last sum is $2^{p}$. By the previous lemma, the right side of this inequality (and thus both sides) is less than or equal to $2^{p}\left(\frac{K^{2} \pi^{2 \alpha}}{2^{2 \alpha p+1}}\right)$ which is equal to $\frac{K^{2} \pi^{2 \alpha}}{2^{p(2 \alpha-1)+1}}$. Now considering the absolute, uniform convergence of the entire Fourier series $\sum_{n=-\infty}^{\infty}|\hat{f}(n)|$, we see that this sum is equal to

$$
|\hat{f}(1)|+|\hat{f}(0)|+|\hat{f}(-1)|+\sum_{p=1}^{\infty} \sum_{2^{p-1}<|n| \leq 2^{p}}|\hat{f}(n)| .
$$

Let $C$ equal $\frac{K^{2} \pi^{2 \alpha}}{2}$. Applying our new version of the inequality from Lemma 3.16 and a geometric sum identity, we get

$$
\begin{aligned}
& |\hat{f}(1)|+|\hat{f}(0)|+|\hat{f}(-1)|+\sum_{p=1}^{\infty} \sum_{2^{p-1}<|n| \leq 2^{p}}|\hat{f}(n)| \\
& \leq|\hat{f}(1)|+|\hat{f}(0)|+|\hat{f}(-1)|+\frac{C}{2^{2 \alpha-1}} \frac{1}{1-2^{2 \alpha-1}} .
\end{aligned}
$$

Note that the geometric sum identity only holds if $2 \alpha-1$ is greater than 0 , or $\alpha>1 / 2$. This bounds the infinite sum in question $\sum_{n=-\infty}^{\infty}|\hat{f}(n)|$, and we can apply Theorem 3.9 to give us the desired result.

This theorem gives us something much stronger than our previous result from Theorem 3.11, requiring only a Hölder condition of order $\alpha>1 / 2$ to guarantee convergence of a function $f$ 's Fourier series to $f$. 


\section{ISOPERIMETRIC INEQUALITy}

Now let's look at one practical application of the material in this paper, the isoperimetric inequality. Prior to stating the theorem we should define a few things:

Definition 4.1. A curve is the image of a function $\gamma$, which is a mapping $\gamma$ : $[a, b] \rightarrow \mathbb{R}^{2}$. For our purposes, a curve must also be continuously differentiable. We denote a curve with the symbol $\Gamma$. A curve is called simple and closed if it does not intersect itself, but its starting and ending points coincide; that is, $\gamma\left(s_{1}\right)=\gamma\left(s_{2}\right)$ if and only if $s_{1}$ and $s_{2}$ are the endpoints $a$ and $b$.

- Length of a curve $\Gamma$ is defined as $l=\int_{a}^{b}\left|\gamma^{\prime}(s)\right| d s$ or $\int_{a}^{b}\left(x^{\prime}(s)^{2}+y^{\prime}(s)^{2}\right)^{1 / 2} d s$ if $\Gamma$ is parametrized by $\gamma s=(x(s), y(s))$.

- Area enclosed by a curve $\Gamma$ is defined using Green's Theorem as $A=$ $\frac{1}{2}\left|\int_{\Gamma}(x d y-y d x)\right|$ or $\frac{1}{2}\left|\int_{a}^{b}\left(x(s) y^{\prime}(s)-y(s) x^{\prime}(s)\right) d s\right|$ (more information on Green's Theorem and its use in calculating area can be found in [2]).

Theorem 4.2. Suppose that $\Gamma$ is a simple closed curve in $\mathbb{R}^{2}$ of length $l$, and let $A$ denote the area of the region enclosed by this curve. Then

$$
A \leq \frac{l^{2}}{4 \pi}
$$

with equality if and only if $\Gamma$ is a circle.

Proof. Without loss of generality, we can assume $x^{\prime}(s)^{2}+y^{\prime}(s)^{2}=1$ for all $s \in$ $[0,2 \pi]$. This is equivalent to letting the length of our curve equal $2 \pi$. Then we see that

$$
\frac{1}{2 \pi} \int_{0}^{2 \pi}\left(x^{\prime}(s)^{2}+y^{\prime}(s)^{2}\right) d s=1 .
$$

Here $x(s)$ and $y(s)$ are $2 \pi$-periodic because they parametrize a closed curve, so we may consider the following Fourier series:

$$
x(s) \equiv \sum a_{n} e^{i n s}, y(s) \equiv \sum b_{n} e^{i n s}, x^{\prime}(s) \equiv \sum a_{n} i n e^{i n s}, y^{\prime}(s) \equiv \sum b_{n} i n e^{i n s}
$$

Note that the series for $x^{\prime}$ and $y^{\prime}$ are such due to Lemma 3.10. We can apply Parseval's Identity to 4.3 by splitting the integral into two integrals:

$$
\begin{gathered}
\frac{1}{2 \pi} \int_{0}^{2 \pi} x^{\prime}(s)^{2} d s+\frac{1}{2 \pi} \int_{0}^{2 \pi} y^{\prime}(s)^{2} d s=\sum_{n=-\infty}^{\infty}\left|a_{n} i n\right|^{2}+\sum_{n=-\infty}^{\infty}\left|b_{n} i n\right|^{2} \\
=\sum_{n=-\infty}^{\infty}|n|^{2}\left(\left|a_{n}\right|^{2}+\left|b_{n}\right|^{2}\right)=1
\end{gathered}
$$

Because $x(s)$ and $y(s)$ are real-valued, we can do the same to our equation defining area, this time with the bilinear form of Parseval's Identity (Lemma 3.8):

$$
\begin{gathered}
A=\pi \frac{1}{2 \pi}\left|\int_{0}^{2 \pi}\left(x(s) y^{\prime}(s)-y(s) x^{\prime}(s)\right) d s\right| \\
=\pi\left|\sum_{n=-\infty}^{\infty} a_{n} \overline{i n b_{n}}-\sum_{n=-\infty}^{\infty} b_{n} \overline{i n a_{n}}\right|=\pi\left|\sum_{n=-\infty}^{\infty} n\left(a_{n} \overline{b_{n}}-b_{n} \overline{a_{n}}\right)\right| .
\end{gathered}
$$


Now we can apply a pair of inequalities that are true for all complex numbers and are easy to verify:

$$
\left|a_{n} \overline{b_{n}}-b_{n} \overline{a_{n}}\right| \leq 2\left|a_{n}\right|\left|b_{n}\right| \leq\left|a_{n}\right|^{2}+\left|b_{n}\right|^{2}
$$

We also have the inequality $|n| \leq|n|^{2}$ because $n \in \mathbb{Z}$, so we may use Equation 4.4 and our area equation to get

$$
A \leq \pi \sum_{n=-\infty}^{\infty}|n|^{2}\left(\left|a_{n}\right|^{2}+\left|b_{n}\right|^{2}\right)=\pi .
$$

This gives us the first part of the theorem. When $A=\pi$, we must have both $|n|=|n|^{2}$ for all $n$ and equality in Equation 4.5. This means our parametric equations look like this:

$$
x(s)=a_{-1} e^{-i s}+a_{0}+a_{1} e^{i s}, y(s)=b_{-1} e^{-i s}+b_{0}+b_{1} e^{i s}
$$

We also know that both equations are real-valued, so $a_{-1}=\overline{a_{1}}$ and $b_{-1}=\overline{b_{1}}$ (because all imaginary parts of the Fourier series will cancel out). Equation 4.4 implies that $2\left(\left|a_{1}\right|^{2}+\left|b_{1}\right|^{2}\right)=1$, and the second inequality in Equation 4.5 then implies that $a_{1}=b_{1}=1 / 2$. We can write $a_{1}=\frac{1}{2} e^{i \alpha}$ and $b_{1}=\frac{1}{2} e^{i \beta}$ and use the first inequality in Equation 4.5 and Euler's Formula, Equation 1.1, to see that $|\sin (\alpha-\beta)|=1$, or $\alpha-\beta=k \pi / 2, k \in \mathbb{Z}$. Applying Euler's Formula again, we see then that

and

$$
x(s)=\frac{1}{2} e^{i(-\alpha-s)}+a_{0}+\frac{1}{2} e^{i(\alpha+s)}=a_{0}+\cos (\alpha+s)
$$

$y(s)=\frac{1}{2} e^{i(-\beta-s)}+b_{0}+\frac{1}{2} e^{i(\beta+s)}=b_{0}+\cos (\beta+s)=b_{0}+\cos \left(\alpha+s-\frac{k \pi}{2}\right)=b_{0} \pm \sin (\alpha+s)$

These equations parametrize a circle, proving the second part of the theorem.

Acknowledgments. I would like to thank my mentor, Max Engelstein, for all of his help, encouragement, and support. His patience and guidance made this paper possible, and I cannot thank him enough. I would also like to thank E. M. Stein and R. Shakarchi for their excellent book on Fourier analysis and acknowledge that this work has been my primary guide in writing this paper. Finally, I'd like to thank Peter May for organizing a fantastic REU and Laszlo Babai for his eye-opening class.

\section{REFERENCES}

[1] Elias M Stein and Rami Shakarchi. Fourier Analysis: An Introduction. Princeton University Press. 2003.

[2] Mary L. Boas. Mathematical Methods in the Physical Sciences. John Wiley and Sons Inc. 2005.

[3] Sergey V. Konyagin. Almost everywhere convergence and divergence of Fourier series. http://www.icm2006.org/proceedings/Vol_II/contents/ICM_Vol_2_66.pdf. 